\title{
Tidal dissipation in creeping ice and the thermal evolution of Europa
}

\author{
Christine McCarthy $^{1 *}$ and Reid F. Cooper ${ }^{2}$
}

${ }^{1}$ Lamont-Doherty Earth Observatory, Columbia University, Palisades, New York, USA

${ }^{2}$ Department of Earth, Environmental and Planetary Sciences, Brown University, Providence, Rhode Island, USA

*corresponding author: mccarthy@1deo.columbia.edu

\section{Abstract}

The thermal and mechanical evolution of Europa and comparable icy satellites - the physics behind creating and sustaining a subsurface water ocean-depends almost entirely on the mechanical dissipation of tidal energy in ice to produce heat, the mechanism(s) of which remain poorly understood. In deformation experiments, we combine steady-state creep and low-frequency, small-strain periodic loading, similar conditions in which tectonics and tidal flexing are occurring simultaneously. The data reveal that the relevant, power-law attenuation in ice (i) is non-linear, depending on strain amplitude, (ii) is independent of grain size, and (iii) exceeds in absorption the prediction of the Maxwell solid model by an order of magnitude. The Maxwell solid model is widely used to model the dynamics of planetary ice shells, so this discrepancy is important. The prevalent understanding of damping in the geophysical context is that it is controlled by chemical diffusion on grain boundaries, which renders attenuation strongly dependent on grain size. In sharp contrast, our results indicate instead the importance of intracrystalline dislocations and their spatial interactions as the critical structural variable affecting dissipation. These dislocation structures are controlled by stress and realized by accumulated plastic strain. Thus, tectonics and attenuation are coupled, which, beyond the icy satellite/subsurface ocean problem, has implications also for understanding the attenuation of seismic waves in deforming regions of the Earth's upper mantle.

\section{Introduction}

Although water ice is one of the most common substances in the solar system, it is also one of the most enigmatic, with much remaining unknown about its viscoelastic properties. To interpret surface features of icy satellites and to model thermal evolution via tidal dissipation, a thorough understanding of the rheological properties of ice and ice-rich mixtures at planetary conditions is needed. Without such experimental data, processes on icy satellites typically have been modeled using a simple Maxwell solid or a 
36 steady-state rheology (e.g., Ojakangas and Stevenson, 1989). However, the disparity

37 between predicted behavior using Maxwell's model and observed behavior from recent

38 satellite-based measurements (e.g., geysers and tectonics) demonstrate the need to refine

39 such models to include the spectrum of mechanical response from elastic to anelastic to

40 viscous (Shoji et al., 2013). To account for significant heat generated by mechanical

41 dissipation of tidal forces, a transient, anelastic response is required. Since microstructure

42 influences transient/anelastic properties and steady-state behavior establishes and sustains

43 microstructure, the overlap of transient and steady-state behavior is important for tidal

44 processes. Further, the ability to extrapolate laboratory data to planetary dynamics

45 requires understanding of the physics of dissipation and how it scales with frequency,

46 grain size, and temperature, as well as with stress and accumulated strain.

47 On Europa, the global stress field within the icy shell includes a diurnal component with

48 a frequency $f=3 \times 10^{-6} \mathrm{~Hz}$ from periodic tidal flexing with a strain amplitude of $\sim 10^{-5}$

49 (Tobie et al., 2003). Additionally the presence of the liquid ocean decouples the shell,

50 resulting in a significantly larger component of tidal stress from reorientation of the tidal

51 bulge relative to the parent body's direction, called nonsynchronous rotation, which has a

52 larger strain amplitude and much larger period $\left(\sim 10^{13} \mathrm{~s}\right)$ (Greenberg et al., 1998). In

53 addition to these two periodic stresses, it is posited that convective overturn can occur

54 within an icy shell creating a stress on the order of tens to hundreds of $\mathrm{kPa}$ (Pappalardo et

55 al., 1998).

56 Here we describe and interpret the results from a series of experiments that measure the

57 anelastic response of polycrystalline ice simultaneously experiencing a cyclic load and a

58 constant steady-state load at realistic stress/strain amplitudes and at frequencies 
59 approaching those of satellite tides. The tests highlight the fact that different mechanisms

60 act at different timescales: the mechanism rate-limiting high-strain creep need not be the

61 same as that providing the relaxation of a small periodic perturbation. The beauty of

62 attenuation is that it can be used as mechanical spectroscopy to see the mechanisms (via

63 small disturbances) shrouded by the steady-state behavior.

\section{2. Experimental Method}

65 In this study deformation experiments were conducted on polycrystalline ice samples. We

66 used three different methods of sample preparation in order to obtain specimens with four

67 distinct grain sizes (Table 1): (1) coarse (C: $d \sim 300 \mu \mathrm{m}$ ); (2) medium (M: $d \sim 150 \mu \mathrm{m}$ ); (3)

68 fine (F: $d \sim 20 \mu \mathrm{m}$ ); and (4) very fine (VF: $d \sim 8 \mu \mathrm{m}$ ). Those methods were: bulk

69 solidification from seeded water to create C samples (McCarthy et al., 2007);

70 nebulization of water with flash freezing followed by sieving to desired particle size and

71 "hot"-pressing to create M and F samples (Goldsby and Kohlstedt, 1997); and ice-II to

72 ice-I phase transformation via a pressure release protocol to create VF samples (Durham

73 et al., 2001). The details of each fabrication method can be found in Appendix A.1.

74 Sample characterization was conducted using a scanning electron microscope (SEM)

75 fitted with a cryogenic preparation station. Secondary electron images (SEI) were taken

76 of fresh fracture surfaces while remaining under vacuum with $\mathrm{T}<100 \mathrm{~K}$ and a low

77 accelerating voltage of $2 \mathrm{kV}$ (Fig. 1). Sublimation rates in the SEM are known to be

78 higher at grain boundaries, ostensibly etching the samples (Cullen and Baker, 2001).

79 Grain size was measured for each of the VF, F, and C samples using the line intercept

80 method on SEM images, with a correction factor of 1.5 (Gifkins, 1970). A total of 7

81 transects and 53 grains were counted from three VF sample images; 24 transects and 252 
82 grains were counted from seven F sample images; and 5 transects and 28 grains were

83 counted from one $\mathrm{C}$ sample image. The grain size errors listed parenthetically in Table 1

84 represent the standard deviation from the mean of these counts. Several samples were

85 examined prior to deformation and after deformation and no discernable grain growth

86 occurred during mechanical testing.

87 Using a commercial servomechanical-actuator testing apparatus that was modified for

88 cryogenic conditions, samples were subjected to a sinusoidally time-varying compressive

89 stress superposed upon a constant, median applied compressive stress. The resulting

90 strain — a steady-state creep strain plus a periodic anelastic strain — was measured with a

91 gravity-fed extensometer that, by specifications, could resolve differential strains of $\varepsilon=$

$925 \times 10^{-7}$; thermal noise limited the strain resolution to $5 \times 10^{-6}$. Complex modulus and

93 attenuation $Q^{-1}$ are measured from the amplitude ratio and the tangent of phase delay $\delta$,

94 respectively, of the peak stress $\sigma$ and peak strain $\varepsilon$. (A detailed description of the

95 apparatus and data analyses are provided in Appendix A.) In this study the tests employed

96 uniaxial loading so that the properties measured were Young's modulus $E$ and attenuation

$97 Q_{E}^{-1}$. In most cases, the median applied stress was $\sigma_{m}=1 \mathrm{MPa}$ (Table 1). For this median

98 stress, and the temperatures and grain sizes of the majority of these samples, the steady-

99 state flow was accomplished by grain boundary sliding that is strain-accommodated by

100 basal dislocation slip (Goldsby and Kohlstedt, 2001), the mechanism thought to be most

101 applicable to tectonic processes in the geophysical ice-shell problem (Barr and Showman,

102 2009). In the case of the coarsest-grain-sized specimens in this study, deformation

103 conditions were instead consistent with dislocation creep. The range of applied stress

104 sinusoids studied was $\sigma_{0}=0.05-0.28 \mathrm{MPa}$ (cyclic strain amplitude $\varepsilon_{0} \approx 5 \times 10^{-6}-3 \times 10^{-5}$ ), 
105 with $0.167 \pm 0.006 \mathrm{MPa}\left(1.7 \times 10^{-5}\right)$ being the amplitude used for most tests (and $0.16 \mathrm{MPa}$

106 the value used for analyses). Testing was conducted in the temperature range $200 \leq \mathrm{T}(\mathrm{K})$

$107 \leq 260$ and over the frequency range $10^{-4}-10^{-1} \mathrm{~Hz}$. Error was determined by performing

108 multiple tests (between three and five, depending on frequency) and plotting the mean

109 value and absolute variation thereof.

\section{3. Experimental Results}

111 The steady-state effective viscosities demonstrated by our specimens are consistent with

112 those from previous experimental studies on similar polycrystalline specimens of ice-I

113 (e.g., Goldsby \& Kohlstedt, 2001). A typical creep curve from initial loading (prior to the

114 periodic loading) is provided in the Appendix (Fig. A2(a)). Figure 2 presents the results

115 of linearity tests, which were confined to the $\mathrm{F}(\sim 20 \mu \mathrm{m})$ specimens and run at $f=0.01$

$116 \mathrm{~Hz}$. The median differential stress for all tests was $\sigma_{m}=1 \mathrm{MPa}$. The material

117 demonstrates a modest strain-amplitude dependence of attenuation, $Q_{E}{ }^{-1} \propto \varepsilon_{0}^{0.39}$, with the

118 response insensitive to temperature, to first order.

119 Attenuation and Young's modulus data for the fine-grained (F) samples as a function of

120 testing temperature are presented in Fig. 3. The data display clear temperature

121 dependence, with, at fixed frequency, decreasing effective modulus and increasing

122 attenuation with increasing temperature. The form of the attenuation spectra (Fig. 3(b)) is

123 a modest power-law relationship with frequency, $Q_{E}^{-1} \propto f^{-\varphi}$, where $\varphi$ increases with

124 temperature from $\sim 0.16$ at $200 \mathrm{~K}$ to $\sim 0.5$ at $250 \mathrm{~K}$. (Data from $260 \mathrm{~K}$ are too scattered to fit

125 responsibly with a line.) Significant and almost total relaxation of the modulus from its

126 high-frequency (i.e., unrelaxed or anharmonic) value $E_{U}$ is observed (Fig. 3(a)). 
127 Determination of activation enthalpy $\left(E_{A}\right)$ for the anelastic response is problematic: the

128 variation of $\varphi$ for different temperature conditions affects significantly the

129 characterization. Activation enthalpy is discerned not by variation of $Q_{E}^{-1}$ with

130 temperature but, rather, by the shift in frequency for a specific attenuation response-

131 consistent with Boltzmann statistics [Nowick and Berry, 1972 (p.458); Cooper, 2002].

132 Working through Figs. 3 and 4 at $Q_{E}^{-1}=1$, for example, reveals a range for $E_{A}$ of 25-85

$133 \mathrm{~kJ} \mathrm{~mol}^{-1}\left(E_{A}=58 \pm 25 \mathrm{~kJ} \mathrm{~mol}^{-1}\right)$; analyses for other values of $Q_{E}^{-1}$ confirm the spread.

134 A comparison of attenuation spectra as a function of grain size and temperature is shown

135 in Fig. 4. The data demonstrate clear temperature dependence with a consistent $\varphi$ value

136 amongst samples at each temperature. In contrast, there is very little - to first order, no-

137 dependence of attenuation on grain size, with the exception that $\mathrm{F}$ samples are slightly

138 more attenuating than other samples.

\section{4. Discussion}

140 The results from our study display an apparent dichotomy in the mechanical behavior.

141 The $Q_{E}^{-1} \propto f^{-\varphi}$ power-law response is associated with strictly Newtonian (linear viscous)

142 behavior, which is very sensitive to grain size, whereas the observed strain amplitude

143 dependence is associated with non-Newtonian (non-linear) behavior; further, the

144 attenuation response is independent of grain size, to first order. The seemingly

145 contradictory nature must be reconciled before extrapolation can occur. A first step is to

146 differentiate between the mechanism dominating the viscous response and the

147 mechanism(s) employed by the system to dissipate energy from a small-in-magnitude,

148 limited-in-duration pulse. These mechanisms need not be the same. The steady state

149 response creates and sets the microstructure that the anelastic perturbation "samples". 
150 Here we will take the individual elements of the response, compare the data to previous

151 studies, and by doing so, parse out an understanding of the attenuation response in an

152 actively deforming material.

153 4.1 Previous studies: Diffusionally accommodated GBS

154 Previous studies have determined a universal anelastic response for polycrystalline 155 materials deforming by Newtonian (diffusional) creep (e.g., Gribb and Cooper, 1998; Tan

156 et al., 2001; Jackson et al., 2002; Sundberg and Cooper, 1998). By keeping deviatoric

157 stresses low and grain size small, these studies ensured that no lattice dislocations were

158 nucleated during testing. Thus, the "attenuation band" behavior, that is, the modest

159 power-law frequency dependence in Fig. 5, is attributed to "diffusionally accommodated

160 grain boundary sliding" whereby a deviatoric stress creates high stress concentrations at

161 grain triple junctions that are alleviated through chemical diffusion at length scales of the

162 grain size or larger (e.g., Gribb and Cooper, 1998; Raj and Ashby, 1971; Raj, 1975;

163 Morris and Jackson, 2009). The relaxation time $\tau$ is not that associated with exponential

164 decay, i.e., as represented by the standard anelastic solid, but rather that associated with 165 deviatoric-stress-effected chemical diffusion:

$$
=\frac{3 \sqrt{3}\left(1{ }^{2}\right) d^{3} k T}{2{ }^{3} E_{U} D_{b}}
$$

167 where $v$ is Poison's ratio, $k$ is Boltzmann's constant, $D_{b}$ is the grain-boundary diffusion 168 coefficient of the rate-limiting ionic species, $\zeta$ is the grain-boundary thickness and $\Omega$ is 169 molecular volume (Raj, 1975). In that case, the physics of this relaxation are identical to 170 that of steady-state creep dominated by grain-boundary diffusion; as a consequence, the 
171 relaxation time can be directly calculated from the unrelaxed modulus and the steady-

172 state, diffusional (Newtonian) viscosity $\left(\eta_{s s}\right)$, as (Gribb and Cooper, 1998)

$$
\frac{10 S S}{E_{U}}
$$

174 Equation (1) provides the "license" for normalizing the power-law, high-temperature-

175 background attenuation spectra by the Maxwell frequency, $f_{M} \equiv 1 / \tau_{M}=M_{U} / \eta_{s s}$, where $M_{U}$

176 is the unrelaxed modulus for the applicable mode of testing (McCarthy et al., 2011;

177 Morris and Jackson, 2009). For polycrystalline materials characterized by a Newtonian

178 steady-state viscosity, such normalization results in a master curve on the $\log Q^{-1}$ v. $\log$

$179\left(f / f_{M}\right)$ plot and a relaxation time $\tau$ that is highly sensitive to grain size: $\tau \propto d^{3}$. The

180 compiled data are compared to a frequency-transformed Andrade creep function, which is

181 described more fully in Appendix C. The similitude demonstrated by this normalization is

182 presented in Fig. 5, which includes numerous datasets for polycrystalline olivine-

183 dominated specimens as well as for polycrystalline organic borneol. The efficacy of the

184 master curve method in identifying the diffusionally-based mechanism provides an

185 excellent framework then to analyze other materials that are not deforming by Newtonian

186 (diffusional) creep. In the similarities and differences we can determine the relative

187 importance of other dissipation mechanisms, namely dislocations.

$188 \quad 4.2$ The importance of subgrains

189 Lattice dislocations, because of their high intrinsic energy, arrange themselves in arrays

190 that constitute subgrain boundaries (e.g., Sutton and Balluffi, 1995 (Ch.2)), the spacing

191 distribution of which depends primarily on the deviatoric stress; temperature only enters

192 consideration with regard to its effect on the unrelaxed shear modulus (Twiss, 1977; Raj 
193 and Pharr, 1986). The array of subgrain boundaries, nominally in a log-normal

194 distribution, facilitates dislocation plasticity in that (a) the distribution of subgrains is

195 self-similar to that for "free" (i.e., mobile) dislocations (Weertman and Weertman, 1983;

196 Stone et al., 2004) and (b) diffusional creep of the subgrains is an integral process in

197 dislocation-effected plasticity, one that acts in kinetic parallel (i.e., independently) with

198 dislocation glide (Stone et al., 2004; Stone, 1991). Thus, for materials with a

199 dislocation/subgrain structure set by a dislocation rheology, as is the case for the

200 specimens in this study, one might replace the grain size in Eq. (1) with the mean

201 subgrain size.

202 We can illustrate the effect of subgrains on attenuation by examining Fig. 5, in which we

203 included attenuation data as a function of normalized frequency for a forsterite single

204 crystal that was previously deformed to steady state in the dislocation creep regime

205 (Gueguen et al., 1989). Application of these data to the figure was done using the

206 observed relationship between applied stress and subgrain size from numerous datasets

207 compiled by Raj and Pharr (1986), in which $d_{S G}=K \mu b / \sigma_{\mathrm{m}}$, where $\mu$ is shear modulus, $b$ is

208 the Burgers vector, and constant $K=23$. The $1600^{\circ} \mathrm{C} / 20 \mathrm{MPa}$ steady-state creep

209 conditions equate to a mean subgrain size in the forsterite crystal of $d_{\mathrm{SG}}=33 \mu \mathrm{m}$. The

210 normalization used the $1200^{\circ} \mathrm{C}$ diffusional creep viscosity measured for forsterite

211 (Thompson et al., 2011), with extrapolations using a grain-size exponent of 3 [i.e., $\eta_{s s} \propto$

$212 d^{3}$ (Coble, 1963; Frost and Ashby, 1982)] and a thermal activation energy of $500 \mathrm{~kJ} \mathrm{~mol}^{-1}$

213 (cf. Hiraga et al., 2010). Using these parameters, and the temperature-dependent

214 unrelaxed modulus (Frost and Ashby, 1982), a $33 \mu \mathrm{m}$ "grain size" produces $f_{M}=9.3 \times 10^{-8}$

$215 \mathrm{~s}^{-1}$ for the $1200^{\circ} \mathrm{C}$ attenuation data and $1.2 \times 10^{-5} \mathrm{~s}^{-1}$ for the $1400^{\circ} \mathrm{C}$ data. The normalized 
216 deformed-single-crystal data collapse nicely onto the attenuation master curve, although

217 much of the data exist in a $f / f_{M}$ range of higher frequency than the others. Similitude

218 with the other studies indicates the same mechanism is acting; our analysis shows that in

219 the deformed single crystal case, it is diffusion at the subgrain boundaries responsible for

220 the response. The critical issue in applying the model in Eq. (1) is identifying the

221 characteristic length for chemical diffusion. In Newtonian aggregates having a steady-

222 state diffusional rheology, that characteristic length is the grain size. As will be explored

223 further below, in materials deforming in steady state via a dislocation rheology, the

224 characteristic diffusion length is the subgrain size.

225 The ice specimens we studied are creeping at steady state in the GBS regime or near its

226 transition to dislocation creep. Significant amounts of the plastic strain at steady state are

227 being accomplished by the motion of dislocations. Consequently, steady-state flow in

228 these regimes is consistent with a subgrain structure, which has been recently

229 characterized both by careful experimental ice-rheology study (Hamann et al., 2007) as

230 well as by observational work of deforming ice from Antarctic drill cores (Weikusat et

231 al., 2009). Comparison of the experimental observations of subgrain distribution in

232 Hamann et al., 2007 with the relationship between subgrain size and stress prediction of

233 Raj \& Pharr (1986) demonstrate a direct correlation. The relationship between stress and

234 subgrain size yields a value of $d_{S G} \approx 37 \mu \mathrm{m}$ for ice deforming at steady state at $1 \mathrm{MPa}$.

235 Combined with the temperature-dependent unrelaxed moduli for ice (Gammon et al.,

236 1983) and a parameterization for diffusional creep of ice (Goldsby and Kohlstedt, 2001;

237 cf. Frost and Ashby, 1982), all of the attenuation data collected in this study have been

238 normalized based on mean subgrain size and plotted with the other attenuation data in 
239 Fig. 5. Two points are obvious: (1) with the exception of the highest-temperature (260K)

240 data for the coarsest-grain-sized material, which exhibit significant experimental scatter,

241 the data for the experiments take the same form as the high-temperature-background

242 "master curve"; (2) the attenuation of the deforming ice aggregates in our experiments is

243 approximately an order of magnitude greater at a given frequency than that of the

244 materials in the master curve. (Note that, although there is no theoretically justified

245 reason to do so, normalizing instead with a GBS or dislocation viscosity also yields a

246 curve significantly offset from the master curve of the other studies. Normalizing instead

247 with the diffusion viscosity for the actual average grain size would shift the VF samples

$248 \sim 1$ order of magnitude closer to the master curve, the F samples would be nearly the

249 same, but the $\mathrm{M}$ and $\mathrm{C}$ samples would shift 2 orders of magnitude farther to the right.)

$250 \quad 4.3$ Dissipation by lattice dislocations

251 The rock physics/materials science understanding of dislocation damping is evolving.

252 Non-linear results such as ours have historically been treated with the dislocation-glide-

253 damping model of Granato \& Lücke (1956a; 1956b; cf. Minster and Anderson, 1981;

254 Karato and Spetzler, 1990; Farla et al., 2012). Their model assumes dislocations pinned

255 by other dislocations and/or by impurities, producing either frequency-dependent

256 attenuation via motion constrained by the pinning-essentially the vibration of

257 dislocations - or frequency-independent attenuation based on dislocation glide after

258 breaking away from pinning. A statistical spatial distribution of pinning points is required

259 to produce a distribution of compliances, but reasonable assumptions for impurities, etc.,

260 cannot replicate the form or frequency of our data: the processes articulated by Granato \&

261 Lücke, for reasonable assumptions of stress and temperature (normalized to modulus and 
262 melting temperature, respectively), apply to frequencies higher than the ones studied in

263 our experiments and, additionally, produce $\varphi \geq 1$ for the frequency-sensitive attenuation

264 response in material that includes an exponential distribution of dislocation pinning

265 lengths. Clearly, the model cannot explain the present results.

266 A way forward in understanding the measured attenuation response takes advantage of

267 recent experimental characterizations of dislocation plasticity in ice. Steady-state flow in

268 ice has been demonstrated to involve intermittent emissions and motions of collections of

269 dislocations - dislocation "bursts" - that have been characterized by acoustic emissions

270 (Miguel et al., 2001; Richeton et al., 2005; Weiss et al., 2015). These studies reveal self-

271 organized, critical (SOC) behavior in deforming ice, identified by an inverse power law

272 relationship between the energy magnitude of a dislocation burst and the frequency of

273 bursts at that magnitude. Such a mechanical result is inconsistent with the textbook

274 understanding of crystalline plasticity based upon the behavior of the "average"

275 dislocation (e.g., Frost and Ashby, 1982). The result, however, is certainly consonant

276 with the dislocation substructure in steady-state creep being statistical in nature and self-

277 similar, as introduced earlier (e.g., Stone et al., 2004; Zaiser and Hähner, 1999). Further,

278 the statistical, SOC nature of plasticity is consistent with Stone's (1991) model for

279 dislocation creep, which is predicated on the equation-of-state approach to plasticity

280 championed by Hart (1970) and demonstrated to hold for all classes of crystalline solids

281 [metallic (e.g., Hart and Solomon, 1973), ionic (e.g., Stone et al., 2004; Lerner et al.,

282 1979) and covalent (e.g., Chiang and Kohlstedt, 1985)]. The model partitions strain

283 between diffusional creep of subgrains and temperature-insensitive (athermal) glide, 284 depending on the exact size of a given subgrain relative to the stress. In thinking about 
285 attenuation, perturbations in stress, those small in magnitude and of limited temporal

286 extent relative to the stress sustaining the steady-state flow, merely "tickle" this self-

287 similar substructure without prompting its adjustment. The predicted anelastic response is

288 linear, i.e., that associated with diffusional transients with the subgrain size defining the

289 characteristic length scale, as introduced above-hence the linear anelastic response of

290 the dislocation-creep-deformed forsterite crystal described earlier and presented in Fig. 5

291 (Gueguen et al, 1989). There is, however, a threshold stress perturbation where mobile

292 dislocations are nucleated/emitted from grain and subgrain boundaries (cf. Gifkins,

293 1976), and within the context of Stone's model (1991), SOC behavior in plasticity, then,

294 can be understood as setting a stress- and temperature-sensitive probability for achieving

295 the threshold and so the emission of dislocations that can glide across one or several

296 subgrains or even the entire specimen (Puthoff, 2005).

297 We can scrutinize Fig. 5 to calculate the energy dissipation by dislocation motion alone.

298 Glide of dislocations is "pure" dissipation: plasticity being a constant-volume process,

299 elastic energy stored in the bonds of a stressed material is converted entirely into heat by

300 the glide motion. Consider, as an example, a datum from the center of our experimental

301 set-a fine-grain-size (F) ice specimen tested at 240K with $\sigma=\sigma_{m} \pm \sigma_{0}=1 \pm 0.16 \mathrm{MPa}$.

302 The material demonstrates a $Q_{E}^{-1}=2$ at $f=3 \times 10^{-3} \mathrm{~Hz}$ and a measured $E=1.3 \mathrm{GPa}$ (Fig.

3033 ; this datum can be located at $Q_{E}^{-1}=2$ and $f / f_{M} \approx 10^{1} \mathrm{~Hz}$ in Fig. 5); thus, $\varepsilon_{0} \cong 9 \times 10^{-4}$

304 and the storage modulus $\left(E^{\prime}=E \cos \delta\right)$ is $0.58 \mathrm{GPa}$. One can compare this to the master

305 curve for the polycrystalline data, where the attenuation is an order-of magnitude lower,

306 i.e., $Q_{E}^{-1}=0.2$. For this master-curve value of $Q_{E}^{-1}$, the storage modulus is estimated as

$3070.97 E_{U}$, (from a Lissajous figure, e.g., Lakes, 1999 (p.68)), which at 240K is 9.2 GPa. For 
308 a half-cycle of loading, with this modulus and $\sigma_{0}=0.16 \mathrm{MPa}$, the stored elastic energy is

$309 W_{s}=115 \mathrm{~J} \mathrm{~m}^{-3}$; thus, following the definition of $Q^{-1}$ as the energy dissipated per radian,

310 i.e.,

$$
Q^{1} \equiv \frac{1}{2}\left(\frac{W_{d}}{W_{s}}\right),
$$

312 where $W_{d}$ is the dissipated energy (e.g., Nowick and Berry, 1972 (p.22), Lakes, 1999

313 (p.76)), the dissipated energy for $Q_{E}^{-1}=0.2$ is $W_{d}=144 \mathrm{~J} \mathrm{~m}^{-3}$. The additional dissipated

314 energy of the actual specimen, with a $Q_{E}{ }^{-1}=2$, then, is $\Delta W_{d}=1296 \mathrm{~J} \mathrm{~m}^{-3}$ and represents

315 a plastic strain of $\sim 8 \times 10^{-4}$.

316 The self-similar microstructural state of the ice in our experiments associated with, the

317 subgrain-size distribution is "set" by the mean stress, $\sigma_{m}$, which for most of our

318 experiments is 1.0 MPa. A distribution of subgrain sizes in a self-similar structure must

319 relate to a distribution of mobile dislocations, which in turn can be characterized by a

320 mean dislocation density, $\rho_{d}$ (Stone et al., 2004). Plastic strain (and energy dissipation) is

321 given by the product of the dislocation density times the magnitude of the Burgers vector

$322\left(b\left[=4.52 \times 10^{-10} \mathrm{~m}\right.\right.$ for basal dislocations in ice $\left.]\right)$ times the average distance traveled by a

323 dislocation $(x)$, i.e., $\varepsilon=\rho_{d} b x$. Application of the strain-energy piezometer, here $\rho_{d}=$

$324\left(\sigma_{m} /\left[3 \mu_{U} b\right]\right)^{2}$ (e.g., Weathers et al., 1979), at $240 \mathrm{~K}$ and with $\sigma_{m}=1 \mathrm{MPa}$ gives $\rho_{d}=$ $3254.2 \times 10^{10} \mathrm{~m}^{-2}$. Thus, the plastic strain of $8 \times 10^{-4}$ can be achieved by the $\rho_{d}$ mobile

326 dislocations gliding by $40 \mu \mathrm{m}$, which is approximately the same as the subgrain size.

327 The result is a satisfying one: a release and glide of the average density of dislocations

328 across the average subgrain size characterizes the additional energy dissipated over a 
329 condition where the modulus is only modestly relaxed. Use of average (mean) quantities

330 is justified in the same spirit as that involved in, e.g., other dissipative, power-law

331 systems (e.g., Weertman and Weertman, 1983; Stone et al., 2004) or in the description of

332 bulk thermodynamic parameters such as system enthalpy. Of course, the same calculation

333 done with data from other frequencies would not be as easily interpreted physically: at

334 lower frequencies, an order of magnitude additional dissipation over the master curve

335 represents significantly more additional dislocation glide while the opposite is true at

336 higher frequency.

337 The first-order lack of dependence of the attenuation response on grain size provokes

338 additional comment. Given that we have explored a grain size range of approximately

339 two orders of magnitude, and that distinctly finer-grain-size material (VF; $\sim 8 \mu \mathrm{m}$ ) shows

340 the same attenuation at lower frequency, an attenuation, too, that matches that of our

341 coarse $(\mathrm{C} ; \sim 300 \mu \mathrm{m})$ material, informs our claim of grain-size independence. One

342 ramification of this argument is that the subgrain size that we used to normalize our

343 attenuation data in Fig. 5-37 $\mu \mathrm{m}$-is coarser than our finest grain size material (VF).

344 Analysis of the relative strain energy densities of dislocations, subgrain boundaries and

345 grain boundaries support the idea that dislocation networks can have scales exceeding the

346 grain size [e.g., Lubarda et al., 1993]; further, plastic flow effected by dislocations can

347 occur in a kinetic regime that doesn't "feel" the grain boundaries [e.g., Crossman and

348 Ashby, 1975]. It remains interesting, however, that the one specimen type having a

349 subgrain size and grain size approximately equal demonstrates the greatest attenuation-

350 albeit not by much, but well within experimental resolution-in these experiments,

351 suggesting as additive the grain-boundary sliding/subgrain boundary sliding/dislocation 
352 emission effects. The additive kinetic responses combined with the fractal distribution of

353 subgrain sizes associated with steady-state creep address, in part, the breadth of activation

354 energies seen in our experiments. The range of values for $E_{A}\left(58 \pm 25 \mathrm{~kJ} \mathrm{~mol}^{-1}\right.$ at $Q_{E}{ }^{-1}=$

355 1) is consistent with dislocation and diffusional mechanisms both being involved in the

356 anelastic response [e.g., Goldsby and Kohlstedt, 2001; cf. Cole and Durell, 2001].

\section{4.4 Application to Geophysical contexts}

358 The findings of this study have implications for both seismic attenuation in the Earth's

359 upper mantle and tidal dissipation on icy satellites. Regions of the upper mantle that

360 display seismic anisotropy are interpreted as being deformed (either actively or in the

361 past) via dislocation creep in response to the tectonic stress. Consistent with the results

362 from a previous study on pre-deformed olivine samples (Farla et al., 2012), our study

363 suggests that seismic attenuation may be enhanced by deformation-induced

364 microstructure. However, additional testing, particularly at normalized frequencies that

365 should be those of the seismic band are needed to accurately scale and predict the

366 magnitude of the effect. The frequencies of tidal loading of some icy satellites, however,

367 are well within the normalized range shown in Fig. 5 (depending on the size and period of

368 the moon). In these cases, direct extrapolation can be performed by determining the stress

369 associated with solid-state convection in the ice shell and calculating from this stress and

370 the mean temperature a characteristic subgrain size. As the temperatures and periodic

371 stress amplitude in our experiments match that of the tidal flexing we calculate the

372 frequency shift of the experimental data. The convection stress is estimated as

$$
\sigma \cong(0.1-0.01) \rho g \alpha_{V}(\Delta T) D
$$


374 where $\rho$ is density $\left(\rho_{\text {icel }}=920 \mathrm{~kg} \mathrm{~m}^{-3}\right), g$ is gravitational acceleration, $\alpha_{V}$ is the coefficient 375 of volumetric thermal expansion $\left(\alpha_{V \text {,icel }}=1.6 \times 10^{-4} \mathrm{~K}^{-1}\right), \Delta T$ is the magnitude of the 376 temperature fluctuations driving convection (or, alternately the difference in temperature

377 from the bottom to top of the convecting layer), and $D$ is the convecting layer thickness

378 (Barr and Showman, 2009, cf. Tobie et al., 2003; Solomatov and Moresi, 2000; Tobie et 379 al., 2005). For Europa, $g=1.314 \mathrm{~m} \mathrm{~s}^{-2}, D=12 \mathrm{~km}$ and $\Delta T=270-230 \mathrm{~K}=40 \mathrm{~K}$ (Tobie et 380 al., 2003). Substituting all of these values yields a convection stress of $\sigma \approx 1$ to $10 \mathrm{kPa}$ 381 and so corresponds with a subgrain size ranging from $d_{S G}=3.7 \mathrm{~cm}(1 \mathrm{kPa})$ to $d_{S G}=0.37 \mathrm{~cm}$ 382 (10 kPa; Raj and Pharr, 1986). As presented earlier, our data, with $\sigma_{m}=1 \mathrm{MPa}$, has a $d_{S G}$ $383=37 \mu \mathrm{m}$. Now that we have estimated a subgrain size, the next task is to extrapolate to icy 384 satellites using a physical approach.

385 Factors like temperature and grain size affect the frequency of the dynamic response 386 (e.g., Nowick and Berry, 1972 (p.458); Cooper, 2002): $f$ is proportional to $\left(d_{S G}\right)^{p}$ $387 \exp \left(E_{A} / \mathrm{R} T\right)$. Thus, the extrapolation for the subgrain size, holding both $T$ and $\sigma_{0}$ constant, 388 is $\left(f_{1} / f_{2}\right)=\left(d_{S G 2} / d_{S G 1}\right)^{p}$. Accordingly, we extrapolate the data for $240 \mathrm{~K}$ (Fig. $3 ; \varphi=0.33$ ) 389 to the subgrain size associated with convection at $\sigma=1 \mathrm{kPa}$ and determine the value of $390 Q_{E}$ at $f=10^{-5} \mathrm{~Hz}$, the tidal flexing frequency. For $p=3$, the $\left(1 \mathrm{kPa}, 10^{-5} \mathrm{~Hz}\right)$ conditions 391 produce $Q_{E}=100\left(Q_{E}{ }^{-1}=0.01\right)$; for $p=2$, one discerns $Q_{E}=10\left(Q_{E}{ }^{-1}=0.10\right)$. This latter 392 value is provocative, perhaps, in that $Q=10$ was a foundational assumption in the 393 Europan heat budget (Ojakangas and Stevenson, 1989).

394 We argue, however, that experimental and theoretical geophysics can and should do

395 better than this. The change in slope of the $\log f \mathrm{v} \cdot \log Q^{-1}$ spectrum in Fig. $3 \mathrm{~b}$ is related 
396 to real physics. It results from the intrinsic viscosity of grain boundaries (e.g., Sundberg

397 and Cooper, 2010; Morris and Jackson, 2009; cf. Gifkins, 1976) or, more likely in the 398 geophysical setting, of subgrain boundaries (e.g., Cooper, 2002). As such, the

399 extrapolated numbers for $Q_{E}^{-1}$ in the previous paragraph are diminished ones, that is, we

400 anticipate greater attenuation in polycrystalline ice at the tidal frequency of $10^{-5} \mathrm{~Hz}$ for an

401 ice shell convecting under a stress of $\sim 1 \mathrm{kPa}$. Additionally stress concentrations in the

402 shell, associated perhaps with features detected and characterized on the surface, become

403 sites for additional heat input. As localized heat input affects local viscosity, mechanical

404 instability and strain heterogeneity within the shell can be anticipated. The crucial

405 scientific insight required to extrapolate laboratory measurements of mechanical

406 dissipation to planetary conditions is a characterization of the materials physics behind

407 the observed $\varphi=\varphi\left(f, T, \sigma_{m}\right)$ relationship and its parameterization for incorporation into

408 models for dynamics in ice shells.

\section{5. Conclusions}

410 It has long been known that frequency, temperature, and pressure affect attenuation.

411 Grain size has also been documented to greatly affect attenuation, but specifically for

412 very fine grain sizes at conditions where the median stress is small, approaching zero.

413 These are conditions that only infrequently conform to geologic settings. This study

414 explored the effect of a persistent creep stress combined with small amplitude

415 perturbations, as would be the case for a seismic wave passing through an actively

416 deforming (tectonic) region in the Earth or for an icy shell experiencing both convective

417 and tidal stresses. Contrary to previous low-stress studies, we find grain size to be

418 unimportant in the attenuation response compared to the deformation-induced subgrain 
419 size. And in polycrystalline ice, additional energy dissipation comes from motion of

420 lattice dislocations. These two effects manifest as a measured attenuation significantly

421 higher than that predicted by a simple Maxwell solid model. Additional understanding

422 about how the attenuation scales with median stress and accumulated strain, a focus of

423 current studies, may elucidate the origin of anomalously high heat fluxes observed on icy

424 satellites.

425

426

427

428

429

430

431

432

433

434

435

436

\section{Acknowledgments}

The authors thank Marc Hesse for assistance with mechanical data reduction, Sara Berglund for help in fabricating samples, Yasuko Takei for many fruitful discussions about anelasticity and scaling, the U.S.G.S. in Menlo Park, CA for use of their cryoS.E.M., and Al Tente and Brian Corkum (both at Brown University) for their technical support with the experiments. The authors also thank $\mathrm{H}$. de Bresser and a second, anonymous reviewer for thorough and helpful formal reviews of this manuscript. This research was supported financially, in part, by grants (to RFC) from the NASA Program in Planetary Geology and Geophysics (NNX06AD67G) and the NSF Program in Geophysics (EAR-1014476). 
McCarthy \& Cooper: Dissipation in creeping ice

References

Andrade END (1910) On the viscous flow in metals, and allied phenomena. Proc R Soc Lond A84: 1-12.

Barr AC \& Showman AP (2009) in Europa, eds Pappalardo RT, McKinnon WB \& Khurana K (University of Arizona Press, Tucson, AZ), pp 405-430.

Berckhemer H, Kampfmann W, Aulbach E \& Schmeling H (1982) Shear modulus and Q of forsterite and dunite near partial melting from forced-oscillation experiments. Phys Earth Planet Inter 29: 30-41.

Chiang S-W \& Kohlstedt DL (1985) Load relaxation studies of germanium. J Mater Sci 20(2): 736-755.

Coble RL (1963) A model for boundary diffusion controlled creep in polycrystalline materials. J Appl Phys 34: 1679-1682.

Cole, D. M. and G. D. Durell (2001), A dislocation-based analysis of strain history effects in ice, Philos. Mag. A, 81, 1849-1872.

Cooper RF (2002) Seismic wave attenuation: Energy dissipation in viscoelastic crystalline solids. Rev Mineral Geochem 51: 253-290.

Crossman, F. W. and M. F. Ashby (1975), The non-uniform flow of polycrystals by grain boundary sliding accommodated by power-law creep, Acta Metall., 23, 425-440.

Cullen D \& Baker I (2001) Observations of impurities in ice. Micros Res Technq 55: 198-207.

Durham WB, Kirby SH \& Stern LA (1997) Creep of water ices at planetary conditions: A compilation. J Geophys Res 102: 16,293-16,302.

Durham WB, Stern LA \& Kirby SH (2001) Rheology of ice I at low stress and elevated confining pressure. J Geophys Res 106(6): 11,031-11,042.

Duval P (1976) Temporary or permanent creep laws of polycrystalline ice for different stress conditions. Ann Geophys 32: 335-350.

Duval P (1978) Anelastic behaviour of polycrystalline ice. J Glaciol 21(85): 621-628.

Farla RJM, Jackson I, Fitz Gerald JD, Faul UH \& Zimmerman ME (2012) Dislocation damping and anisotropic seismic wave attenuation in earth's upper mantle. Science 336: 332-335.

Frost HJ \& Ashby MF (1982) Deformation-Mechanism Maps: The Plasticity and Creep of Metals and Ceramics, (Pergamon Press, Oxford, UK), pp 166.

Gammon PH, Kiefte H, Clouter MJ \& Denner WW (1983) Elastic constants of artificial and natural ice samples by brillouin spectroscopy. J Glaciol 29(103): 433-460.

Gifkins RC (1970) Optical Microscopy of Metals, (Elsevier, New York), pp 208.

Gifkins RC (1976) Grain-boundary sliding and its accommodation during creep and superplasticity. Metall Trans A 7A: 1225-1232. 
McCarthy \& Cooper: Dissipation in creeping ice

Goldsby DL \& Kohlstedt DL (1997) Grain boundary sliding in fine-grained ice I. Scripta Mater 37: 1399-1406.

Goldsby DL \& Kohlstedt DL (2001) Superplastic deformation of ice: Experimental observations. J Geophys Res 106(B6): 11017-11030.

Granato A \& Lücke K (1956) Application of dislocation theory to internal friction phenomena at high frequencies. J Appl Phys 27(7): 789-805.

Granato A \& Lücke K (1956) Theory of mechanical damping due to dislocations. J Appl Phys 27: 583-593.

Greenberg R, et al (1998) Tectonic processes on Europa: Tidal stresses, mechanical response and visible features. Icarus 135: 64-78.

Gribb TT \& Cooper RF (1998) Low-frequency shear attenuation in polycrystalline olivine: Grain boundary diffusion and the physical significance of the Andrade model for viscoelastic rheology. J Geophys Res 103: 27267-27279.

Gueguen Y, Darot M, Mazot P \& Woirgard J (1989) Q-1 of forsterite single crystals. Phys Earth Planet Inter 55: 254-258.

Hamann I, Weikusat C, Azuma N \& Kipfstuhl S (2007) Evolution of ice crystal microstructure during creep experiments. J Glaciol 53: 479-489.

Hart EW (1970) A phenomenological theory for plastic deformation of polycrystalline metals. Acta Metall 21: 295-307.

Hart EW \& Solomon HD (1973) Load relaxation studies of polycrystalline high-purity aluminum. Acta Metall 21(3): 295-307.

Hiraga T, Miyazaki T, Tasaka M \& Yoshida H (2010) Mantle superplasticity and its selfmade demise. Nature 468: 1091-1094.

Jackson I, Faul UH \& Skelton R (2014) Elastically accommodated grain-boundary sliding: New insights from experiment and modeling. Phys Earth Planet Inter 228: 203-210.

Jackson I, FitzGerald JD, Faul UF \& Tan BH (2002) Grain-size-sensitive seismic-wave attenuation in polycrystalline olivine. J Geophys Res 107(B12): 2360doi:10.1029/2001JB001225.

Karato S-i \& Spetzler HA (1990) Defect microdynamics in minerals and solid-state mechanisms of seismic wave attenuation and velocity dispersion in the mantle. Rev Geophys 28: 399-421.

Lakes RS (1999) Viscoelastic Solids, (CRC Press, Boca Raton, FL), pp 476.

Lee JA \& Cooper RF (1997) Internal friction/attenuation in a beta-spodumene glassceramic. J Am Ceram Soc 80: 2917-2928.

Lerner I, Chiang S-W \& Kohlstedt DL (1979) Load relaxation studies of four alkali halides. Acta Metall 27: 1187-1196.

Lubarda, V. A., J. A. Blume, and A. Needleman (1993), An analysis of equilibrium dislocation distributions, Acta Metall. Mater., 41, 625-642. 
McCarthy \& Cooper: Dissipation in creeping ice

McCarthy C (2009) The microstructures and creep and attenuation behaviors of ice-I and Ice/Hydrate eutectic aggregates at planetary conditions. Ph.D. Dissertation, Brown University (Providence, RI).

McCarthy C, Cooper RF, Kirby SH, Rieck KD \& Stern LA (2007) Solidification and microstructures of binary ice-I/hydrate eutectic aggregates. Am Mineral 92: 15501560.

McCarthy C, Takei Y \& Hiraga T (2011) Experimental study of attenuation and dispersion over a broad frequency range: 2 . the universal scaling of polycrystalline materials. J Geophys Res 116: B09207.

Meyer DW, Cooper RF \& Plesha ME (1993) High-temperature creep and the interfacial mechanical response of a ceramic-matrix composite. Acta Metall Mater 41(11): 3157-3170.

Miguel M, Vespignani A, Zapperi S, Weiss J \& Grasso J (2001) Intermittent dislocation flow in viscoplastic deformation. Nature 410(6829): 667-671.

Minster JB \& Anderson DL (1981) A model of dislocation-controlled rheology for the mantle. Philos Trans R Soc Lond A 299: 319-356.

Morris SJS \& Jackson I (2009) Implications of the similarity principle relating creep and attenuation in finely grained solids. Mater Sci Engr A 521-522: 124-127.

Nowick AS \& Berry BS (1972) Anelastic Relaxation in Crystalline Solids, (Academic Press, San Diego, CA), pp 677.

Ojakangas GW \& Stevenson DJ (1989) Thermal state of an ice shell on Europa. Icarus 81(2): 220-241.

Pappalardo, RT et al (1998) Geological evidence for solid-state convection in Europa's ice shell. Nature 391: 365-367.

Puthoff J (2005) Mesoscale modeling of dislocation bursts during high-temperature plastic deformation. MSc Thesis, University of Wisconsin-Madison, (Madison, WI).

Raj R (1975) Transient behavior of diffusion-induced creep and creep rupture. Metall Trans A 6A: 1499-1590.

Raj R \& Ashby MF (1971) On grain boundary sliding and diffusional creep. Metall Trans 2: 1113-1127.

Raj SV \& Pharr GM (1986) A compilation and analysis of data for the stress dependence of the subgrain size. Mater Sci Engr 81(1-2): 217-237.

Richeton T, Weiss J \& Louchet F (2005) Breakdown of avalanche critical behaviour in polycrystalline plasticity. Nat Mater 4(6): 465-469.

Shoji, D., H. Hussman, K. Kurita, and F. Sohl (2013) Ice rheology and the tidal heating of Enceladus. Icarus 226, 10-19.

Solomatov VS \& Moresi LN (2000) Scaling of time-dependent stagnant lid convection: Application to small-scale convection on earth and other terrestrial planets. J Geophys Res 105(B9): 21795-21817. 
McCarthy \& Cooper: Dissipation in creeping ice

553 Stone DS (1991) Scaling laws in dislocation creep. Acta Metall Mater 39(4): 599-608.

554 Stone DS, Plookphol T \& Cooper RF (2004) Similarity and scaling in creep and load relaxation of single-crystal halite $(\mathrm{NaCl})$. J Geophys Res 109: B12201, doi:10.1029/2004JB003064.

Sundberg M \& Cooper RF (2010) A composite viscoelastic model for incorporating grain boundary sliding and transient diffusion creep; correlating creep and attenuation responses for materials with a fine grain size. Philos Mag 90: 2817-2840.

Sutton AP \& Balluffi RW (1995) Interfaces in Crystalline Materials, (Clarendon PressOxford, Oxford, United Kingdom), pp 819.

Takei Y, Karasawa F \& Yamauchi H (2014) Temperature, grain size, and chemical controls on polycrystal anelasticity over a broad frequency range extending into the seismic range. J Geophys Res 119(7): 5414-5443.

Tan BH, Jackson I \& Fitz Gerald JD (2001) High-temperature viscoelasticity of finegrained polycrystalline olivine. Phys Chem Minerals 28(9): 641-664.

Tatibouet J, Perez J \& Vassoille R (1986) High-temperature internal friction and dislocations in ice Ih. J Physique 47: 51-60.

Tatibouet J, Perez J \& Vassoille R (1987) Study of grain boundaries in ice by internal friction measurement. J Physique 48: 197-203.

Thompson E, Dillman AM, Hansen LN \& Kohlstedt DL (2011) Contribution of grain boundary sliding to diffusion creep of forsterite. Abstract \#T52B-03, Presented at the American Geophysical Union Fall Meeting, San Francisco, CA, 9 December 2011

Tobie G, Choblet G \& Sotin C (2003) Tidally heated convection: Constraints on Europa's ice shell thickness. J Geophys Res 108(E11): 5124, doi:10.1029/2003JE002099.

Tobie G, Mocquet A \& Sotin C (2005) Tidal dissipation within large icy satellites: Applications to Europa and Titan. Icarus 177: 534-549.

Twiss RJ (1977) Theory and applicability of a recrystallized grain size paleopiezometer. Pure Appl Geophys 115: 227-244.

Weathers MS, Bird JM, Cooper RF \& Kohlstedt DL (1979) Differential stress determined from deformation-induced microstructures of the Moine Thrust Zone. J Geophys Res 84: 7495-7509.

Weertman J \& Weertman JR (1983) in Physical Metallurgy (Third Edition), eds Cahn JW \& Haasen P (Elsevier Science Publishers B.V., Amsterdam), pp 1309-1340.

Weikusat I, Kipfstuhl S, Faria SH, Azuma N \& Miyamoto A (2009) Subgrain boundaries and related microstructural features in EDML (Antarctica) deep ice core. J Glaciol 55: 461-472.

Weiss, J., W. Ben Rhouma, T. Richeton, S. Dechanel, F. Louchet, and L. Truskinovsky (2015) From Mild to Wild Fluctuations in Crystal Plasticity, Physical Review Letters, 114, 105504.

Zaiser M \& Hähner P (1999) The flow stress of fractal dislocation arrangements. Mater Sci Engr A 270(2): 299-307. 
McCarthy \& Cooper: Dissipation in creeping ice

Table 1: Experimental conditions for dynamic testing and Maxwell frequency $f_{M}$ used for scaling in Fig. 4

\begin{tabular}{|c|c|c|c|c|c|c|}
\hline $\begin{array}{c}\text { Grain size } \\
\quad(\mu m)\end{array}$ & $\begin{array}{c}\text { Grain size } \\
\text { designation }\end{array}$ & $T(K)$ & $\begin{array}{c}\text { Median } \\
\text { stress } \\
\sigma_{m}(\mathrm{MPa}) \\
\end{array}$ & $\begin{array}{c}\text { Stress } \\
\text { Amplitude } \\
\sigma_{0}(\mathrm{MPa}) \\
\end{array}$ & Frequency range $f(H z)$ & $\begin{array}{c}f_{M}\left(s^{-1}\right)^{*} \\
\varepsilon \eta_{s s}\end{array}$ \\
\hline $8.4(1.8)$ & VF & 220 & 1.0 & 0.168 & $2.5 \times 10^{-4}-2.5 \times 10^{-2}$ & $2.19 \times 10^{-5}$ \\
\hline $8.4(1.8)$ & VF & 240 & 1.0 & 0.167 & $10^{-3}-5 \times 10^{-2}$ & $3.08 \times 10^{-4}$ \\
\hline $8.4(1.8)$ & VF & 260 & 0.78 & 0.168 & $2.5 \times 10^{-4}-10^{-2}$ & $1.75 \times 10^{-3}$ \\
\hline 19.9(3) & $F$ & 200 & 1.0 & 0.171 & $7.5 \times 10^{-4}-10^{-1}$ & $9.27 \times 10^{-7}$ \\
\hline 19.9(3) & $F$ & 220 & 0.96 & 0.168 & $2.5 \times 10^{-4}-5 \times 10^{-2}$ & $2.02 \times 10^{-5}$ \\
\hline 19.9(3) & $\mathrm{F}$ & 220 & 1.07 & 0.168 & $10^{-1}$ & $2.52 \times 10^{-5}$ \\
\hline 19.9(3) & $\mathrm{F}$ & 240 & 0.87 & 0.172 & $10^{-3}-2.5 \times 10^{-1}$ & $2.33 \times 10^{-4}$ \\
\hline 19.9(3) & $F$ & 240 & 0.96 & 0.166 & $10^{-4}$ & $2.89 \times 10^{-4}$ \\
\hline 19.9(3) & $\mathrm{F}$ & 240 & 1.04 & 0.173 & $2.5 \times 10^{-4}-7.5 \times 10^{-3}$ & $3.33 \times 10^{-4}$ \\
\hline 19.9(3) & $\mathrm{F}$ & 250 & 1.0 & 0.169 & $7.5 \times 10^{-3}-7.5 \times 10^{-2}$ & $9.57 \times 10^{-4}$ \\
\hline 19.9(3) & $\mathrm{F}$ & 260 & 0.84 & 0.168 & $10^{-3}-5 \times 10^{-2}$ & $2.02 \times 10^{-3}$ \\
\hline $100-180$ & $\mathrm{M}$ & 240 & 1.0 & 0.165 & $5 \times 10^{-4}-7.5 \times 10^{-2}$ & $3.08 \times 10^{-4}$ \\
\hline $100-180$ & $M$ & 260 & 1.0 & 0.165 & $10^{-2}-10^{-1}$ & $2.87 \times 10^{-3}$ \\
\hline $300(60)$ & $\mathrm{C}$ & 240 & 1.0 & 0.165 & $10^{-3}-7.5 \times 10^{-3}$ & $3.08 \times 10^{-4}$ \\
\hline $300(60))$ & $\mathrm{C}$ & 260 & 1.27 & 0.163 & $10^{-3}-7.5 \times 10^{-3}$ & $4.63 \times 10^{-3}$ \\
\hline $300(60)$ & $\mathrm{C}$ & 260 & 1.44 & 0.162 & $7.5 \times 10^{-3}-10^{-2}$ & $5.96 \times 10^{-3}$ \\
\hline $300(60)$ & $\mathrm{C}$ & 260 & 1.69 & 0.162 & $5 \times 10^{-4}-5 \times 10^{-3}$ & $8.27 \times 10^{-3}$ \\
\hline
\end{tabular}

$594 * \eta_{\mathrm{sS}}$ is the diffusion creep viscosity estimated using Goldsby \& Kohlstedt (2001) at the given T and a subgrain size 595 calculated from Raj \& Pharr (1986) at $\sigma_{m}$. 
Figure 1. SEM/SEI images of water ice-I made via three different fabrication techniques: (a) very fine-grained VF $(8.4 \pm 1.8 \mu \mathrm{m})$ made by pressure release method which results in subdivision of originally larger, spherical grains; (b) fine-grained $F(19.9 \pm 3.0 \mu \mathrm{m})$ prepared by droplet solidification, in which triple junctions and pronounced grain boundary troughs can be seen; and (c) coarse-grained C $(300 \pm 60 \mu \mathrm{m})$ grown slowly by bulk solidification in test tubes. A fourth grain size, medium-grained $\mathrm{M}(<180 \mu \mathrm{m})$ was also fabricated using the droplet solidification method (but a larger sieve), but was not imaged. Grain size for specimens $\mathrm{M}$ is estimated from the sieve size. A dashed white line in each figure roughly outlines a single grain.

Figure 2. Measured attenuation vs. strain amplitude (the latter calculated using the known applied stress normalized by the temperature dependent unrelaxed Young's modulus). Data represent fine grain samples $(\mathrm{F})$ tested at $f=0.01 \mathrm{~Hz}$. Where error bars are not apparent, it is because they are less than the size of the symbols. The slope of the $260 \mathrm{~K}$ line is 0.32 , the slope of the $240 \mathrm{~K}$ line is 0.46 and the slope of the $220 \mathrm{~K}$ line is 0.38 . The $200 \mathrm{~K}$ data show considerable scatter.

Figure 3. Measured (a) modulus dispersion and (b) attenuation, as functions of frequency and temperature for fine grain samples $(\mathrm{F} ; \sim 20 \mu \mathrm{m})$ made by droplet solidification/hot-pressing. The reciprocating stress amplitude $\sigma_{0}$ was constant in all the tests, but the median stress $\sigma_{m}$ varied from $0.83 \mathrm{MPa}(260 \mathrm{~K}$ data) to $1.04 \mathrm{MPa}$ (see Table 1$)$.

Figure 4. Measured attenuation vs. frequency as a function of grain size at $220 \mathrm{~K}, 240 \mathrm{~K}$, and $260 \mathrm{~K}$. The solid gray line is a simple approximation of the $\mathrm{F}(\sim 20 \mu \mathrm{m})$ data at $240 \mathrm{~K}$ (gray circles). The dashed line is an extrapolation of this data to predict the response of $\mathrm{C}$ samples $(\sim 300 \mu \mathrm{m})$ based on an inverse cube of grain size relationship for the viscosity term of the Maxwell frequency, which is the prevailing theory for the grain size dependence of attenuation in this frequency range. The predicted attenuation is significantly lower than that measured for any of the grain sizes at this temperature, demonstrating that in our study attenuation is independent of grain size, to first order.

Figure 5. Measured $Q^{-1}$ for polycrystalline materials at various temperatures and grain sizes. All data have been normalized by the Maxwell frequency $\left(f_{\mathrm{M}}=M_{U} / \eta_{s s}\right)$ where $\eta_{s s}$ is the steadystate diffusion creep viscosity. Data for olivine are from Gueguen, 1989 (1200 and $1400^{\circ} \mathrm{C}$; pre-deformed forsterite single crystal) Gribb and Cooper, $1998\left(1200\right.$ and $1250^{\circ} \mathrm{C} ; d=3$ $\mu \mathrm{m})$, Jackson et al., $2002\left(1200^{\circ} \mathrm{C} ; d=2.9 \mu \mathrm{m}\right)$, Tan et al., $2001\left(1200^{\circ} \mathrm{C} ; d=23.6 \mu \mathrm{m}\right)$, and Berckhemer et al., $1982\left(1400^{\circ} \mathrm{C} ; d=1 \mathrm{~mm}\right.$ synthetic forsterite); data for borneol is from McCarthy et al., $2001\left(20-50^{\circ} \mathrm{C} ; d=3-22 \mu \mathrm{m}\right)$. In ice data from this study, $\eta_{s s}$ is estimated diffusion creep viscosity from Goldsby and Kohlstedt, 2001 assuming a grain size consistent with the stress-dependent subgrain size and temperature dependent moduli. The gray line is the Andrade model using $\eta_{s s}$ estimate assuming diffusion creep in ice $\left(1.4 \times 10^{16}\right.$ 
Pa s), a Young's modulus of $9 \mathrm{GPa}$, and best fit parameters for $\beta$ and $\psi\left(4.5 \times 10^{-12}\right.$ and 0.25 , respectively; cf. SI) and normalized by the Maxwell frequency.

Figure A1. Apparatus used for compression-compression cyclic loading experiments (schematic). Three features to note are (1) the cryostat, which uses circulating nitrogen gas as the temperature regulator, (2) the free-floating, gravity-fed LVDT extensometer that sits outside the cold chamber and is accessible within an open cage, and (3) the load train made entirely of low-thermal-conductivity, high-stiffness Macor ${ }^{\mathrm{TM}}$ fluorophlogopite glassceramic.

Figure A2. (a) Typical creep curve obtained after initial loading of sample $\left(\sigma_{\mathrm{m}}=1 \mathrm{MPa}\right)$, prior to cyclic loading. (b) Typical raw data for $\mathrm{Q}_{\mathrm{E}}^{-1}$ experiments (gray). A numerical algorithm fits curves (black dashed line) to both the load and displacement data and so provides an output with the phase difference. The approach isolates the mechanical testing frequency from other, higher-frequency noise associated with, e.g., temperature fluctuation. For both figures, the data shown were collected while testing very fine grain sized samples (VF) at $\mathrm{T}$ $=240 \mathrm{~K}$. For $(\mathrm{b}), f=0.01 \mathrm{~Hz}$, the applied median stress was $\sigma_{\mathrm{m}}=1 \mathrm{MPa}$, and the periodic stress amplitude was $\sigma_{0}=0.16 \mathrm{MPa}$. 


\section{A.1. Sample fabrication.}

659 We used three different methods of sample preparation in order to obtain specimens with four

660 distinct grain sizes. Coarse grain size samples (C) were created by bulk solidification. Pre-scored 661 test tubes, $1 \mathrm{~cm}$ ID and $6.4 \mathrm{~cm}$ long, were filled with distilled water and placed upright in a 662 polystyrene holder that included a well. Liquid nitrogen $(77 \mathrm{~K})$ was poured into the well while the 663 entire holder rested within a freezer $(253 \mathrm{~K})$. Solidification of the sample proceeded from the 664 test-tube bottom at a rate of approximately $20 \mu \mathrm{m} \mathrm{s}^{-1}$. Specimens were sectioned from the middle 665 of the test tube. The $\mathrm{C}$ samples fabricated with this method displayed grains with an average size 666 of $300 \pm 60 \mu \mathrm{m}$ (Fig. 1c and Table 1 of the text proper).

667 The second method of sample fabrication was droplet solidification followed by hot pressing 668 (Goldsby and Kohlstedt, 1997). Distilled water was nebulized into air by a Bernoulli-effect 669 nozzle with orifice diameter $\sim 0.5 \mathrm{~mm}$. The nebulization nozzle siphoned the water at a rate of $670 \sim 50 \mathrm{~mL} \mathrm{~h}^{-1}$ and sprayed the mist directly into a reservoir of liquid nitrogen; the droplets

671 traversed in air a distance of $\sim 1 \mathrm{~cm}$ before entering the liquid nitrogen bath. The result was a 672 solid/liquid nitrogen slurry that was subsequently sieved to collect ice grains of a desired size.

673 Once the liquid nitrogen fully evaporated, this ice powder was "hot" pressed at $196 \mathrm{~K}\left(T / T_{m}=\right.$ $6740.72)$ and $100 \mathrm{MPa}$ for $2 \mathrm{~h}$ in an actively evacuated $(\sim 0.1 \mathrm{kPa})$, stainless steel cylindrical die.

675 This sample preparation method yielded a dense cylindrical specimen with uniform grain size. M 676 samples having a grain size $\leq 180 \mu \mathrm{m}$ and F samples with a grain size of $19.9 \pm 3.0 \mu \mathrm{m}$ (Fig. 1b 677 and Table 1) were prepared by this method. 
679 The final method of sample fabrication utilized the ice-II-to-ice-I transformation in a pressure

680 release protocol described by Durham et al.(1997; 2001), in which samples initially prepared by

681 droplet solidification/hot-pressing, described above, were transformed to ice-II by holding them

682 at $\mathrm{P}=\sim 250 \mathrm{MPa}$ for $5 \mathrm{~min}$. The pressure was then quickly released, which causes rapid

683 nucleation of ice-I at the grain boundaries. After the pressure release, samples were held at 100

684 MPa for another two hours. Since kinetics of nucleation are fast and kinetics of crystal growth

685 are sluggish, the VF prepared by this method exhibited small and uniform grains that were

$686 \quad 8.4 \pm 1.8 \mu \mathrm{m}$ (Fig. 1a and Table 1).

687 All fabrication methods resulted in cylindrical test specimens that were approximately $10 \mathrm{~mm}$ in

688 diameter. The ends of specimens were shaved smooth with a razor blade. Lengths of specimens

689 ranged from 14 to $16 \mathrm{~mm}$.

690 A.2. Apparatus, mechanical testing and data analysis protocol.

691 In this study, Young's modulus and attenuation of polycrystalline ice-I were measured in

692 compressive cyclic loading tests (Lee and Cooper, 1997) using a servomechanical-actuator

693 testing apparatus (Model 1361 Load Frame and Model 8500 Digital Servo Control, Instron

694 Corp.) that was modified for cold-temperature work. The apparatus, as shown schematically in

695 Fig. A1, has several custom-designed/fabricated features including a circulating air (nitrogen

696 gas) cryostat, a gravity-fed extensometer located outside the cryostat (Fig. A1 inset), and a fully

697 ceramic (Macor $\left.{ }^{\mathrm{TM}}\right)$ load train.

698 A sinusoidally varying stress about a constant median differential stress was applied in load

699 control and the resulting displacement was measured with the extensometer, which employs a

700 linear variable differential transformer (LVDT); load, displacement and temperature data were 
701 recorded on a computer via LabView. In all cases, the cryostat and load train were brought to the

702 desired temperature and held there for at least one hour. The sample was then placed in position

703 without load and allowed to equilibrate for 1-2 h. The compressive median differential stress was

704 then applied and the sample allowed to creep for $1-2 \mathrm{~h}$, sufficient to achieve a nominal steady-

705 state (as evidenced in monitoring displacement as a function of time) before the periodic loading

706 commenced (Fig. A2a). Approximately six cycles were run for each testing frequency, which,

707 depending on how many frequencies were measured, totalled between 5 and $9 \mathrm{~h}$ of testing. Prior

708 to and after testing, samples were stored in dry ice $(\mathrm{T}=195 \mathrm{~K})$. Based on the work of Goldsby

709 and Kohlstedt $(1997 ; 2011)$, we expect no microcracking to have occurred in the VF, F, or M

710 samples. In their study, samples made via the same protocols were deformed at comparable

711 stresses to much larger strains in both the $n=1.8$ and $n=2.4$ regimes using an ambient pressure

712 apparatus. The deformed samples in this parallel study were transparent and therefore free of

713 cavities or microcracks. Although post-deformation microscopy was performed on one C sample

714 and no evidence of microcracking was observed, we cannot definitively rule it out in the coarse-

715 grain sized samples.

716 Typical raw experimental data are shown in Fig. A2b, in which time has been zeroed arbitrarily.

717 The monotonic downward trend of the displacement data is a result of steady-state creep

718 associated with the applied median differential stress, $\sigma_{m}=1 \mathrm{MPa}$. Sinusoids were fitted to the

719 collected data using a Levenberg-Marquardt algorithm, seeking parameters consistent with 95\%

720 confidence intervals. The Young's modulus and the phase difference between the applied load

721 and the linear displacement were calculated from the phase and amplitude parameters of the

722 fitted curves. 
723 The error in experimental determination of attenuation in sub-resonant tests like the ones pursued

724 here is primarily in measuring the phase lag $\delta$ separating the applied stress and achieved strain

$725\left(Q^{-1}=\tan \delta\right)$. Other sources of error such as strain resolution of the LVDT and temperature

726 fluctuation are not expected to be significant compared with the error in measuring $\delta$. For these

727 experiments, the error in measuring $\delta$ was determined using both the confidence intervals

728 established by the algorithm and by performing multiple tests (three-to-five, depending on the 729 frequency).

730 Our interest is in characterizing "intrinsic" attenuation, that is, the mechanical loss of a volume

731 of material that is uniformly distorted. As long as the material responds as linear viscoelastic,

732 meaning that the attenuation is not a function of strain amplitude, then the attenuation measured

733 in the experiments is identical to the intrinsic attenuation. As indicated in the Experimental

734 Results section of the text proper, however, and as detailed below, the viscoelastic response of

735 our ice specimens was modestly non-linear. As a consequence, we pursued a theoretical analysis

736 to discern a correction factor between the attenuation we measured and that which is intrinsic

737 (documented in McCarthy, 2009). We determined that the correction-corresponding to our

738 testing geometry and to the modest degree of characterized non-linearity—is quite small, $\sim 6 \%$,

739 which is barely larger than the size of symbols on our attenuation figures and, too, well within

740 experimental error. For this reason, we report in the text proper (and here) the attenuation values

741 directly measured in the experiments.

\section{Appendix B. Non-linearity of Viscoelastic Response.}

743 The attenuation response revealed a very modest nonlinearity- $Q_{E}{ }^{-1} \propto \varepsilon_{0}^{0.39}$ for the range of 744 cyclic strain amplitude explored (Fig 2). In the absence of cracking, which is the case in our 
745 experiments, non-linear response is associated with the presence and motion of lattice

746 dislocations (e.g., Karato and Spetzler, 1990; Nowick and Berry, 1972 [Ch. 11]), although, as

747 explained in the text proper, most experimental and theoretical characterizations of dislocation-

748 effected attenuation in crystalline materials have emphasized conditions at distinctly higher

749 frequencies and lower homologous temperatures than are active in Earth/planetary situations,

750 particularly for water ice.

751 An exception is the work of Tatibouet and colleagues $(1986 ; 1987)$ who studied low-frequency

752 attenuation in ice single crystals, engineered bicrystals and polycrystalline aggregates. For

753 experiments on single crystals (described as "undeformed") and using frequencies in the range

$75410^{-3} \leq f(\mathrm{~Hz}) \leq 1$ and strain amplitudes in the range $2 \times 10^{-5} \leq \Delta \varepsilon_{0} \leq 7.5 \times 10^{-5}$, the authors

755 identified behavior similar to that described here: modest non-linearity where attenuation was

756 proportional to $\varepsilon_{0}{ }^{q}$ where at $10^{-2} \mathrm{~Hz} q$ ranged from $\sim 0.1$ at $250 \mathrm{~K}$ to $\sim 0.2$ at $270 \mathrm{~K}$. For isothermal

757 conditions, however, the non-linearity exponent $q$ increased significantly as the frequency was

758 lowered. Non-linearity was not addressed explicitly in the bicrystal or polycrystalline

759 experiments. Nevertheless, plastically deforming the bicrystals before testing their attenuation

760 response demonstrated the same behavior of modestly nonlinear response that was, too,

761 frequency dependent. While the authors characterized the behavior predicated on dislocation

762 climb and glide dynamics - either of lattice dislocation in the single crystals or of intrinsic grain

763 boundary dislocations in the case of bicrystals and polycrystals - the sensitivity of the high-

764 temperature background characterized in their experiments is at least equally well considered by

765 the combination of chemical diffusion and dislocation emission and glide that we articulate in the 766 text proper. 
768 In Fig. 5 of the text, the gray line behind the master curve experimental data is a frequency-

769 transformation of the Andrade solid model, which incorporates elastic, anelastic and plastic

770 responses (Andrade, 1910):

771

$$
J(t)=\frac{(t)}{=} J_{U}+t+\frac{t}{S S}
$$

772 where $J_{U}$ is the unrelaxed compliance, $\beta$ is a constant $(\beta<<1), t$ is time, $\psi$ ranges from $1 / 4$ to $1 / 2$

773 and $\eta_{\mathrm{ss}}$ is the steady-state viscosity. The Andrade model was first applied to ice by Duval (1976;

774 1978). The storage and loss components of the Andrade model's complex compliance take the

775 forms:

$$
\begin{aligned}
& J_{1}(\quad)=J_{U}+(1+) \quad \cos \left(\frac{}{2}\right) \text { and } \\
& J_{2}(\quad)=(1+) \quad \sin \left(\frac{}{2}\right)+\frac{1}{S S},
\end{aligned}
$$

778 respectively, where $\omega$ is angular frequency $(\equiv 2 \pi f)$ and $\Gamma$ is the gamma function. The Andrade

779 model shown in Fig. 5 is normalized by the Maxwell frequency using an estimated viscosity for

780 grain boundary diffusion creep in ice $\left(1.4 \times 10^{16} \mathrm{~Pa}\right.$ s; Goldsby and Kohlstedt, 2001) and the best-

781 fit parameters for $\beta$ and $\psi\left(4.5 \times 10^{-12}\right.$ and 0.25 , respectively). As the figures show, the shape of

782 the high temperature background, attributed to the diffusion accommodated grain-boundary

783 relaxation mechanism, is generally well captured by the Andrade function. Data generally

784 deviate from the Andrade model at high-normalized frequency where it is considered that 
McCarthy \& Cooper: Dissipation in creeping ice

785 another mechanism is dominating the anelastic response (Sundberg and Cooper, 2010; Jackson et

786 al, 2014; Takei et al., 2014). 

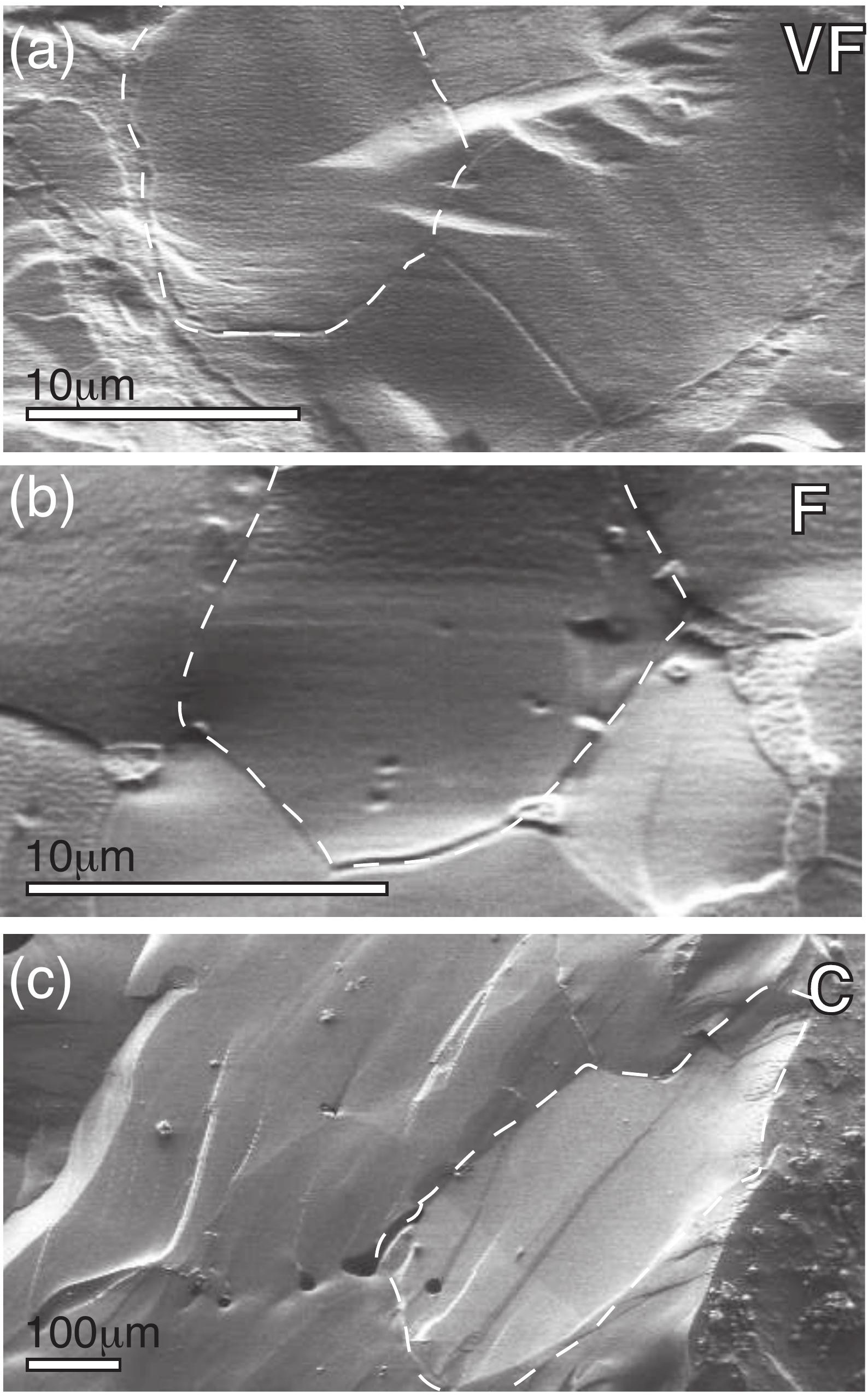

Figure 1

(a)

(b)

1
$=1$ 

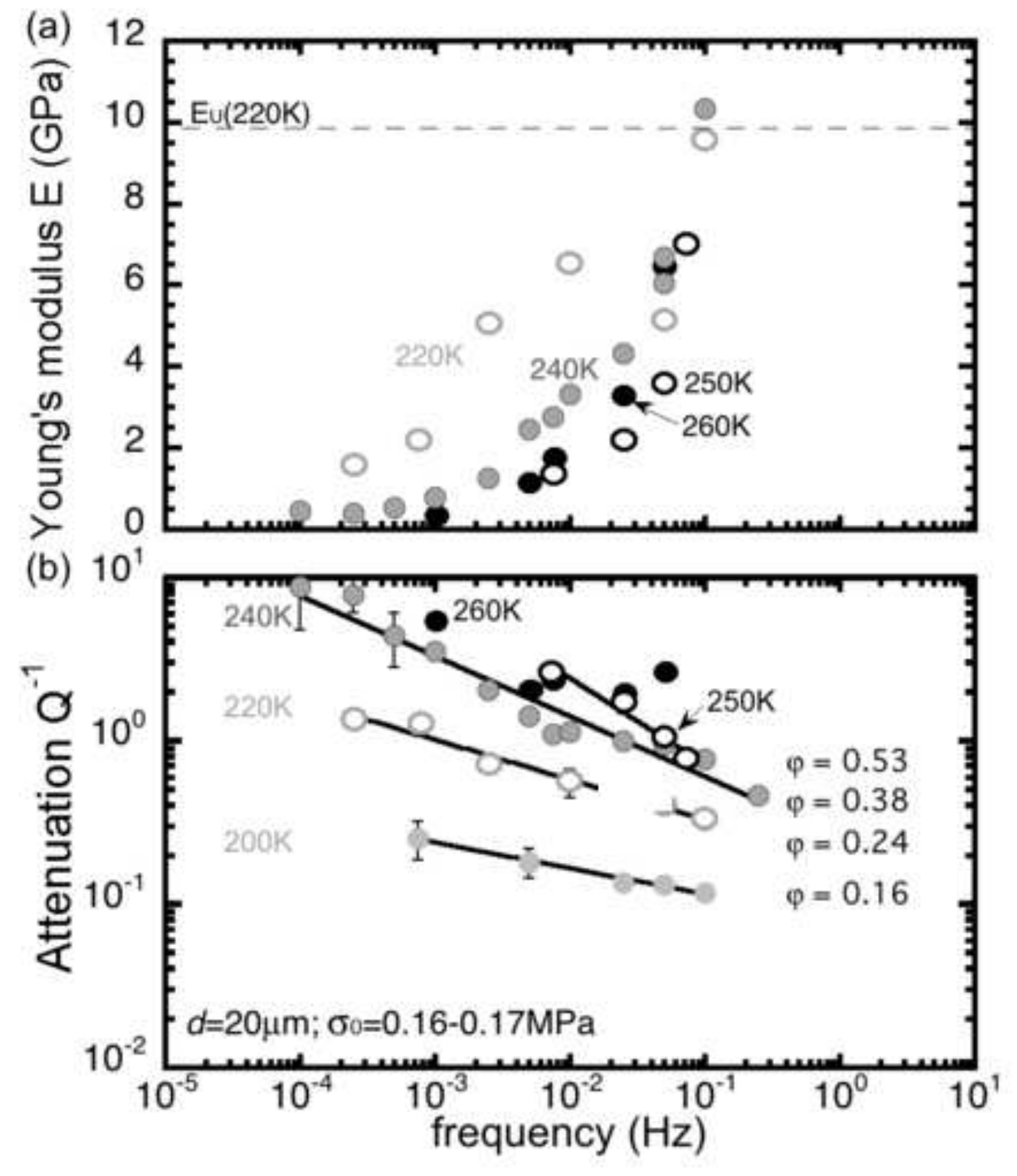

Figure 3 


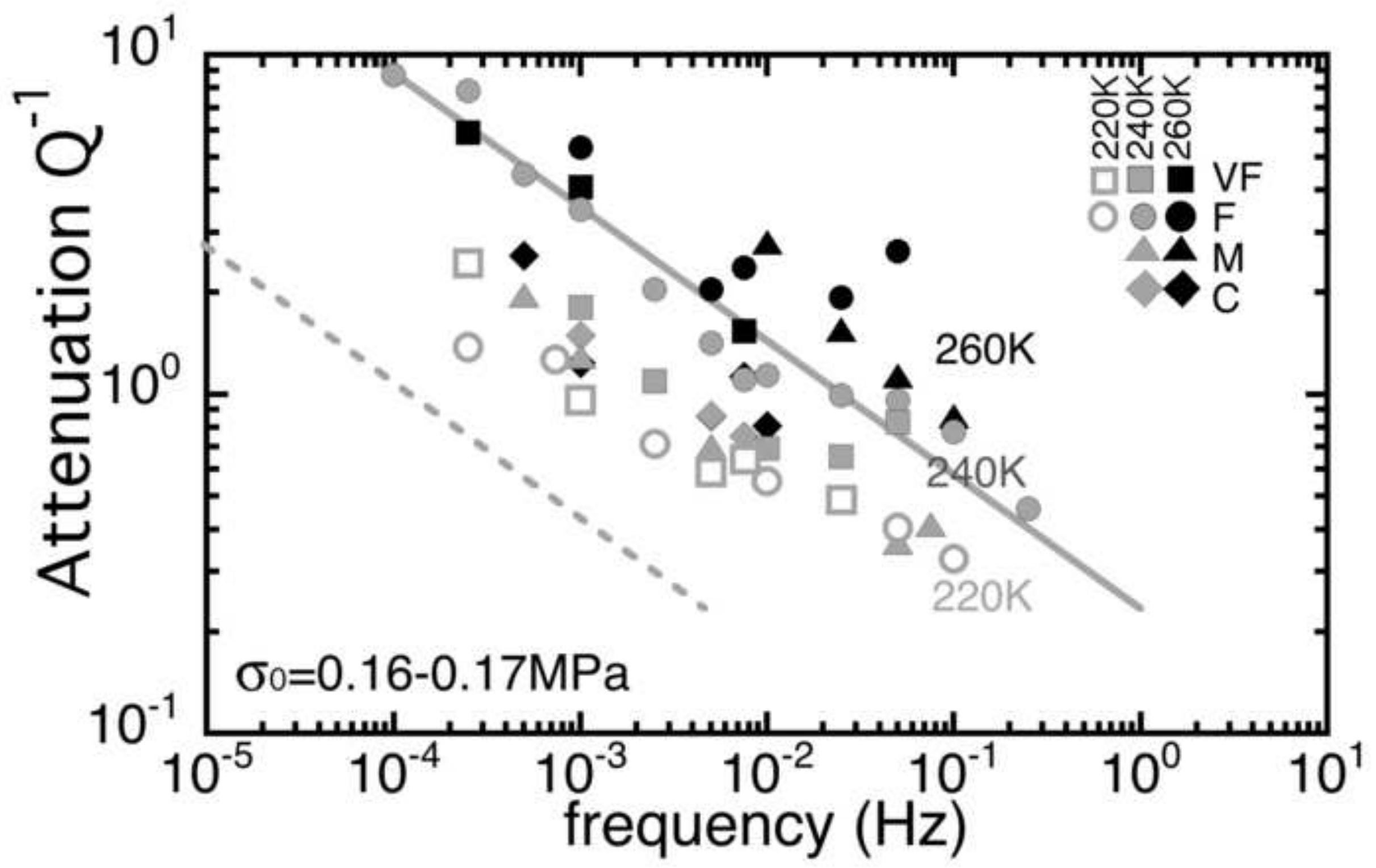




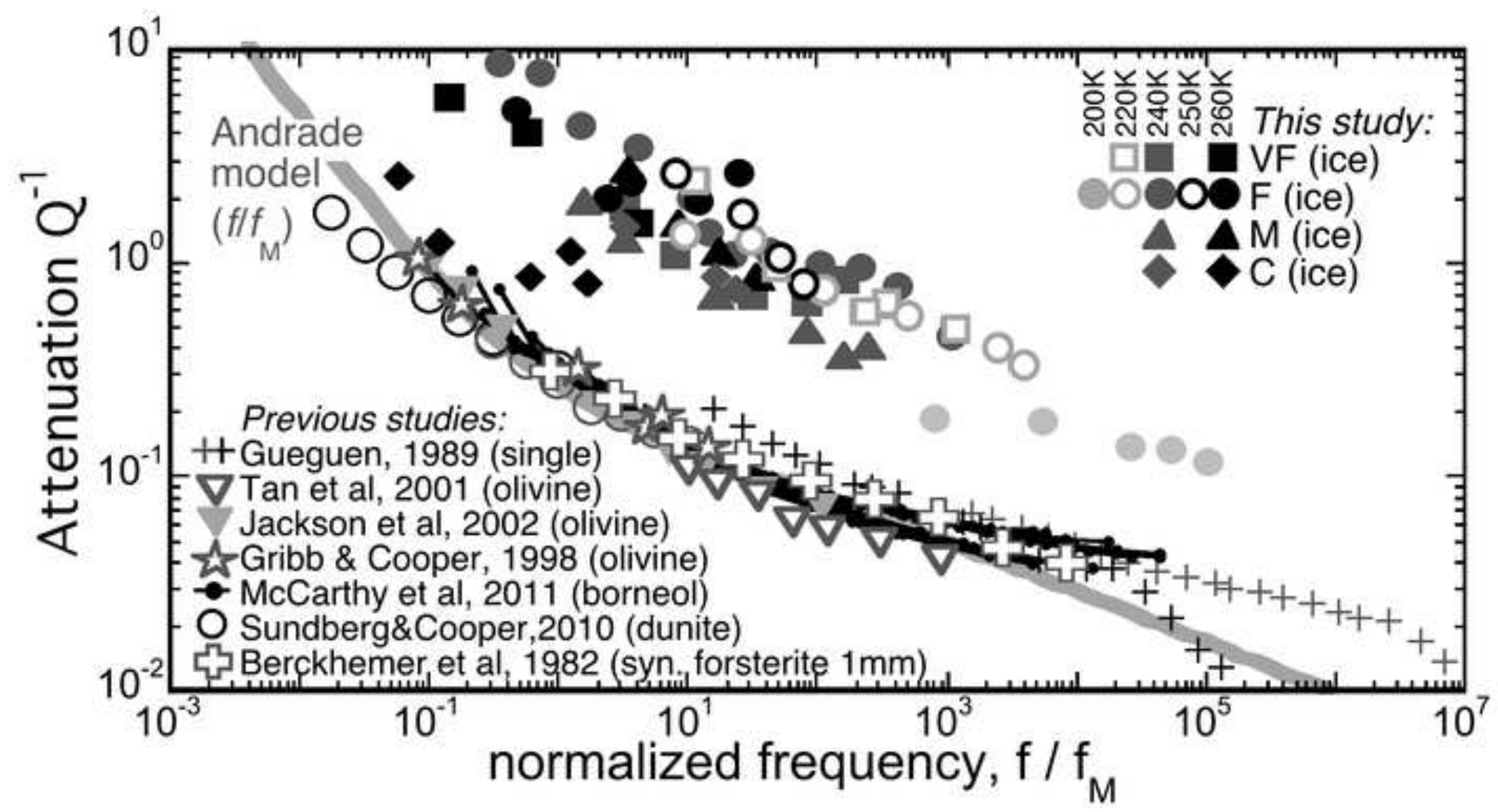




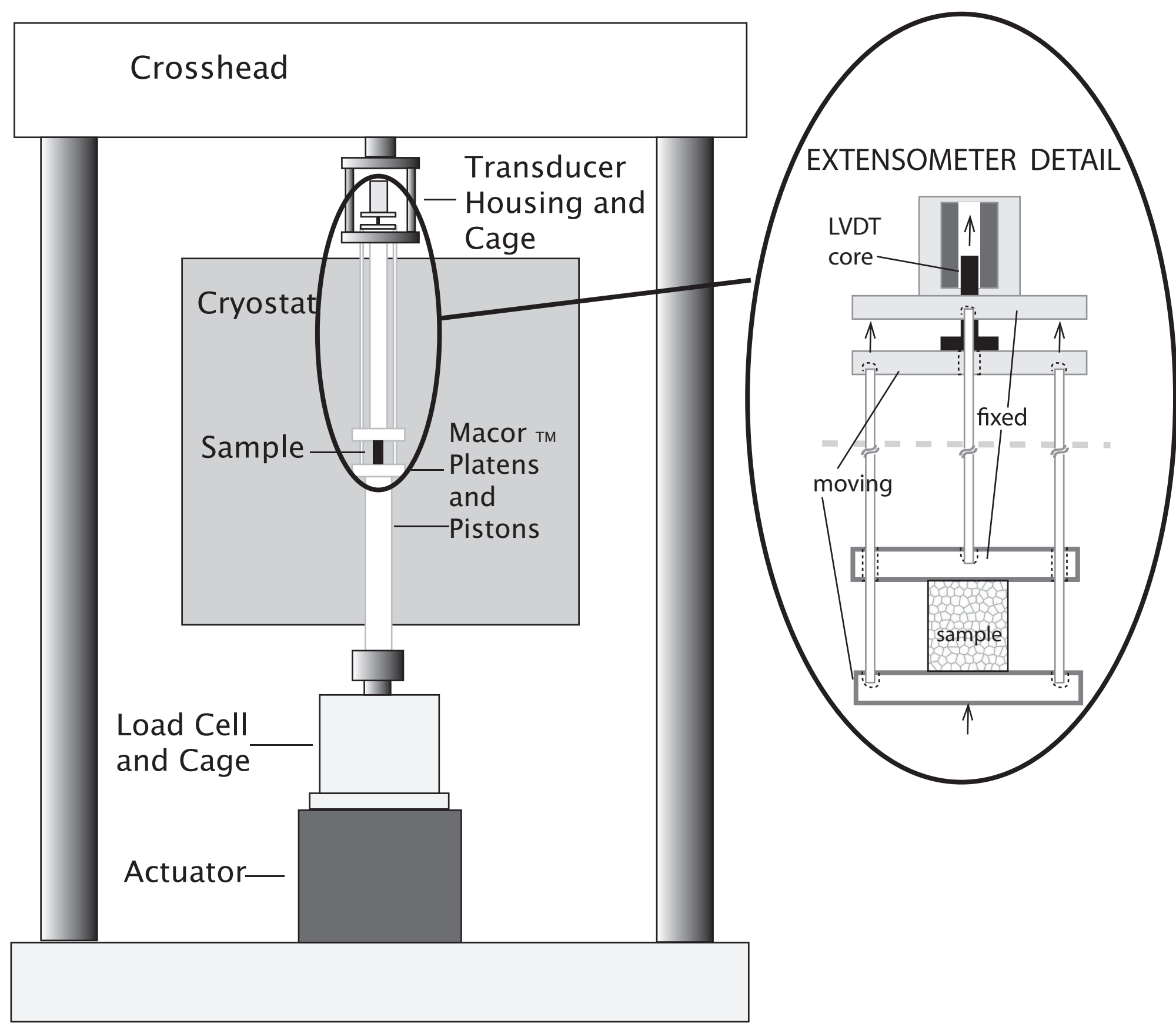



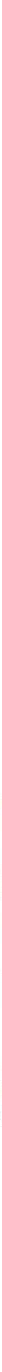